\title{
A THEOREM ON MULTIPLICATIVE ARITHMETIC FUNCTIONS
}

\section{HUBERT DELANGE}

1. Introduction. The following result is contained in a theorem that we proved in a previous paper: ${ }^{1}$

Let $f$ be a real or complex valued multiplicative arithmetic function.

Suppose that $|f(n)| \leqq 1$ for every $n$ and that, as $x$ tends to infinity,

$$
\sum_{p \leq x} f(p) \log p=\rho x+o[x], 2
$$

where $\rho$ is a constant other than 1 .

Then, as $x$ tends to infinity, $\sum_{n \leqq x} f(n)=o[x]$.

The proof of the theorem used a classical tauberian theorem of Hardy and Littlewood.

It is indeed possible to prove the above result without using any tauberian theorem. Besides, this new method enables us to obtain a more precise result. We shall actually prove here the following theorem.

THEOREM. Let $f$ be a bounded multiplicative arithmetic function. Suppose that, as $x$ tends to infinity,

$$
\sum_{p \leq x} f(p) \log p=\rho x+O[x L(x)],
$$

where $\rho$ is a constant $\neq 1$ and $L$ a positive function defined for $x \geqq 1$ and nonincreasing, and $L(x)=o[1]$.

Set $L^{*}(x)=\int_{1}^{x}(L(t) / t) d t(x \geqq 1)$.

Then, as $x$ tends to infinity,

$$
\begin{aligned}
\frac{1}{x} \sum_{n \leq x} f(n) & =O\left[\frac{L^{*}(x)}{\log x}\right] \quad \text { if } \rho=0 \text { or } \quad \text { R } \rho<0, \\
& =O\left[(\log x)^{R_{\rho-1}} \int_{2}^{x} \frac{L^{*}(t)}{t(\log t)^{R+1}} d t\right] \quad \text { if } \rho \neq 0
\end{aligned}
$$

and $R \rho \geqq 0$.

Received by the editors April 26, 1966.

1 Un théorème sur les fonctions arithmétiques multiplicatives et ses applications, Ann. Sci. Ecole Norm. Sup. (3) 78 (1961), 1-29.

2 Throughout this paper $p$ ranges over the primes while $m, n, q$ range over the positive integers.

A sum which contains no term is zero and a product which has no factor is one. 
It is to be noticed that $|\rho|$ must be $\leqq 1$ and, since $\rho \neq 1$, R $\rho$ must be $<1$.

In fact the hypothesis that $f$ be bounded obviously implies that for every $\epsilon>0$ there can be at most a finite number of $p$ 's for which $|f(p)| \geqq 1+\epsilon$, and this in turn implies

$$
\limsup _{x \rightarrow+\infty} \frac{1}{x}\left|\sum_{p \leqq x} f(p) \log p\right| \leqq 1 .
$$

It is also obvious that the theorem implies the above quoted result for, if (1) holds, we have (2) with

$$
L(x)=\underset{u \geqq x}{\operatorname{l.u} . b .}\left|\frac{1}{u} \sum_{p \leqq u} f(p) \log p-\rho\right| .
$$

The idea of trying to improve this result originates from a remark of J. P. Tull, and some points are due to him as indicated in footnotes.

We end the paper by an example of application of the theorem.

2. We need some lemmas.

2.1. Lemma 1. Let $L(t)$ be a real positive function defined for $t \geqq 1$ and nonincreasing.

Then, for $x \geqq 1$,

$$
\sum_{n \leq x} \frac{1}{n} L\left(\frac{x}{n}\right) \leqq L(1)+\int_{1}^{x} \frac{L(t)}{t} d t .
$$

LEMMA 2. $\sum_{m \leqq x ; p^{2} / m} \log p=O[x]$.

The proofs are easy and we leave them to the reader.

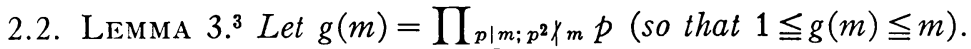

Then $\sum_{m \leqq x} \log (m / g(m))=O[x]$.

Proof. This follows from

$$
\sum_{m \leq x} \log \frac{m}{g(m)}=\sum_{m \leqq x ; p^{2} / m} \log p+\sum_{m \leqq x ; p^{q} / m ; q>1} \log p .
$$

2.3. Lemma $4 .^{4}$ Let $f$ be a bounded multiplicative arithmetic function. Set $\theta_{f}(x)=\sum_{p \leqq x} f(p) \log p$. Then

${ }^{3}$ This lemma is due to J. P. Tull.

4 This lemma is due to J.P. Tull and is an improvement of the preliminary theorem in our paper quoted in footnote 1 . There we supposed $|f(n)| \leqq 1$ for every $n$ and in the conclusion we had $o[1]$ instead of $O[1 / \log x]$ although the method of proof could yield this just as well. 


$$
\frac{1}{x} \sum_{n \leqq x} f(n)=\frac{1}{x \log x} \sum_{n \leqq x} f(n) \theta_{f}\left(\frac{x}{n}\right)+O\left[\frac{1}{\log x}\right] .
$$

Proof. Let $F(x)=\sum_{n \leqq x} f(n)$. Then we have

$$
\sum_{n \leq x} f(n) \log n=F(x) \log x-\int_{1}^{x} \frac{F(t)}{t} d t=F(x) \log x+O[x],
$$

which yields

$$
\frac{1}{x} F(x)=\frac{1}{x \log x} \sum_{n \leqq x} f(n) \log n+O\left[\frac{1}{\log x}\right] .
$$

Therefore we have only to prove that

$$
\sum_{n \leq x} f(n) \log n=\sum_{n \leq x} f(n) \theta_{f}\left(\frac{x}{n}\right)+O[x] .
$$

Now

$$
\begin{aligned}
& \sum_{n \leq x} f(n) \theta_{f}\left(\frac{x}{n}\right)=\sum_{n \preceq \leq x} f(n) f(p) \log p \\
& =\sum_{n p \leqq x ; p \chi_{n}} f(n p) \log p+\sum_{n p \geqq x ; p \nmid \chi^{n}} f(n) f(p) \log p \\
& =\sum_{m \leqq x ; p \mid m ; p^{2} \chi_{m}} f(m) \log p+\sum_{m \leqq x ; p^{2} \chi_{m}} f\left(\frac{m}{p}\right) f(p) \log p \\
& =\sum_{m \leqq x} f(m) \log m-\sum_{m \leqq x} f(m) \log \frac{m}{g(m)} \\
& +\sum_{m \leq x: p^{2} \mid m} f\left(\frac{m}{p}\right) f(p) \log p
\end{aligned}
$$

and the desired result follows by Lemmas 2 and 3 since, if $|f(n)| \leqq M$ for every $n$,

$$
\left|\sum_{m \leqq x} f(m) \log \frac{m}{g(m)}\right| \leqq M \sum_{m \leqq x} \log \frac{m}{g(m)}
$$

and

$$
\left|\sum_{m \leq x ; p^{2} \mid m} f\left(\frac{m}{p}\right) f(p) \log p\right| \leqq M^{2} \sum_{m \leqq x ; p^{2} \mid m} \log p .
$$

3. Proof of the theorem.

3.1. If $|f(n)| \leqq M$ for every $n$, we have 


$$
\begin{aligned}
\left|\sum_{n \leqq x} f(n) \theta_{f}\left(\frac{n}{x}\right)-\rho x \sum_{n \leqq x} \frac{f(n)}{n}\right| & =\left|\sum_{n \leqq x} f(n)\left[\theta_{f}\left(\frac{x}{n}\right)-\rho \frac{x}{n}\right]\right| \\
& \leqq M \sum_{n \leqq x}\left|\theta_{f}\left(\frac{x}{n}\right)-\rho \frac{x}{n}\right| .
\end{aligned}
$$

But there exists a positive $K$ such that

$$
\left|\theta_{f}(x)-\rho x\right| \leqq K x L(x) \text { for } x \geqq 1 .
$$

Thus we get

$$
\left|\sum_{n \leqq x} f(n) \theta_{f}\left(\frac{x}{n}\right)-\rho x \sum_{n \leqq x} \frac{f(n)}{n}\right| \leqq M K x \sum_{n \leqq x} \frac{1}{n} L\left(\frac{x}{n}\right)
$$

and it follows by Lemma 1 that

$$
\sum_{n \unlhd x} f(n) \theta_{f}\left(\frac{x}{n}\right)=\rho x \sum_{n \unlhd x} \frac{f(n)}{n}+O\left[x L^{*}(x)\right] .
$$

By Lemma 4 this yields

$$
\frac{1}{x} \sum_{n \unlhd x} f(n)=\frac{\rho}{\log x} \sum_{n \leqq x} \frac{f(n)}{n}+O\left[\frac{L^{*}(x)}{\log x}\right] .
$$

3.2. Now set as in the proof of Lemma $4 F(x)=\sum_{n \leqq x} f(n)$. We have

$$
\sum_{n \leq x} \frac{f(n)}{n}=\frac{1}{x} F(x)+\int_{1}^{x} \frac{F(t)}{t^{2}} d t=\int_{1}^{x} \frac{F(t)}{t^{2}} d t+O[1] .
$$

Thus (3) gives

$$
\frac{1}{x} F(x)=\frac{\rho}{\log x} \int_{1}^{x} \frac{F(t)}{t^{2}} d t+O\left[\frac{L^{*}(x)}{\log x}\right] .
$$

If we define $G(x)$ for $x>1$ by

$$
G(x)=(\log x)^{-\rho} \int_{1}^{x} \frac{F(t)}{t^{2}} d t,
$$

we see that $G$ is continuous for $x>1$ and differentiable for all nonintegral values of $x$ with

$$
\begin{aligned}
G^{\prime}(x) & =\frac{1}{x(\log x)^{\rho}}\left[\frac{F(x)}{x}-\frac{\rho}{\log x} \int_{1}^{x} \frac{F(t)}{t^{2}} d t\right] \\
& =O\left[\frac{L^{*}(x)}{x(\log x)^{(\operatorname{li}+1}}\right] \text { by (4). }
\end{aligned}
$$


This implies

$$
G(x)=O\left[\int_{2}^{x} \frac{L^{*}(t)}{t(\log t)^{R \rho+1}} d t\right]
$$

that is

$$
\int_{1}^{x} \frac{F(t)}{t^{2}} d t=O\left[(\log x)^{R \rho} \int_{2}^{x} \frac{L^{*}(t)}{t(\log t)^{R \rho+1}} d t\right]
$$

which with (4) yields

$$
\frac{1}{x} \sum_{n \leq x} f(n)=\rho O\left[(\log x)^{R \rho-1} \int_{2}^{x} \frac{L^{*}(t)}{t(\log t) R \rho+1} d t\right]+O\left[\frac{L^{*}(x)}{\log x}\right] .
$$

3.3. To complete the proof it suffices to show that

1. If $R \rho<0$,

$$
\int_{2}^{x} \frac{L^{*}(t)}{t(\log t)^{R \rho+1}} d t=O\left[\frac{L^{*}(x)}{(\log x)^{R \rho}}\right]
$$

2. If $R \rho \geqq 0$,

$$
L^{*}(x)=O\left[(\log x) \operatorname{R\rho } \int_{2}^{x} \frac{L^{*}(t)}{t(\log t)^{\operatorname{R}+1}} d t\right]^{5}
$$

3.3.1. The first assertion is obvious for $L^{*}$ is increasing.

3.3.2. If $R \rho \geqq 0$ we have for $x>4$

$$
\int_{2}^{x} \frac{L^{*}(t)}{t(\log t)^{R p+1}} d t \geqq L^{*}\left(x^{1 / 2}\right) \int_{x^{1 / 2}}^{x} \frac{d t}{t(\log t)^{R_{p+1}}}=C \frac{L^{*}\left(x^{1 / 2}\right)}{(\log x)^{R_{p}}},
$$

where

$$
\begin{aligned}
C & =\left(2^{R_{\rho}}-1\right) / R \rho \quad \text { if } R \rho>0, \\
& =\log 2 \text { if } R \rho=0 .
\end{aligned}
$$

Moreover it is easy to see that $L^{*}(x) \leqq 2 L^{*}\left(x^{1 / 2}\right)$.

Thus if $R \rho \geqq 0$ we have for $x>4$

$$
\int_{2}^{x} \frac{L^{*}(t)}{t(\log t)^{R p+1}} d t \geqq \frac{C}{2} \cdot \frac{L^{*}(x)}{(\log x) R^{\rho}} .
$$

4. Remark. If we assume $\int_{1}^{+\infty}(L(t) / t) d t<+\infty$, the conclusion of the theorem obviously becomes

s This is due to J. P. Tull. 


$$
\begin{aligned}
\frac{1}{x} \sum_{n \leqq x} f(n) & =O\left[\frac{1}{\log x}\right] \quad \text { if } \rho=0 \text { or } R \rho<0, \\
& =O\left[\frac{\log \log x}{\log x}\right] \quad \text { if } \rho \neq 0 \text { and } R \rho=0, \\
& =O\left[(\log x)^{\rho-1}\right] \quad \text { if } R \rho>0 .
\end{aligned}
$$

5. Application. We shall now give an example of application of our theorem.

Let $\omega(n)$ be the number of prime divisors of $n$.

If $f(n)=z^{\omega(n)}$ where $z$ is any complex number whose modulus is $\leqq 1$, then $f$ is a multiplicative function and $|f(n)| \leqq 1$ for every $n$.

Moreover $\sum_{p \leq x} f(p) \log p=z \theta(x)$, where $\theta(x)=\sum_{p \leqq x} \log p$.

If we know that

$$
\theta(x)=x+O\left[x /(\log x)^{\alpha}\right] \quad \text { with } \alpha>1,
$$

we can apply our theorem when $z \neq 1$, with $\rho=z$ and $L(t)$ $=1 /(1+\log t)^{\alpha}$.

By the above remark we conclude that, for $|z| \leqq 1$ and $z \neq 1$,

$$
\begin{aligned}
\frac{1}{x} \sum_{n \leqq x} z^{\omega(n)} & =O\left[\frac{1}{\log x}\right] \quad \text { if } R z<0 \text { or } z=0, \\
& =O\left[\frac{\log \log x}{\log x}\right] \quad \text { if } R z=0 \text { and } z \neq 0, \\
& =O\left[(\log x)^{R^{2-1}}\right] \quad \text { if } R z>0 .
\end{aligned}
$$

Now let $q$ be any integer $>1$ and $r$ any integer.

Denote by $\nu_{q, r}(x)$ the number of $n$ 's $\leqq x$ for which $\omega(n) \equiv r(\bmod q)$. It is plain that

where $\gamma=\exp [2 \pi i / q]$.

$$
\nu_{q, r}(x)=\frac{1}{q} \sum_{j=0}^{q-1}\left\{\gamma^{-j r} \sum_{n \leqq x} \gamma^{j \omega(n)}\right\},
$$

Since, for $1 \leqq j \leqq q-1$, $R \gamma^{j} \leqq \cos (2 \pi / q)$, we obtain

$$
\begin{aligned}
\nu_{q, r}(x)-\frac{x}{q} & =O[x / \log x] \quad \text { if } q \leqq 3, \\
& =O[x \log \log x / \log x] \quad \text { if } q=4, \\
& =O\left[x(\log x)^{-2} \sin ^{2}(\pi / q)\right] \quad \text { if } q>4 .
\end{aligned}
$$

' See e.g. H. Delange, Sur des formules dîes d Atle Selberg, Bull. Sci. Math (2) 83 (1959), 101-111.

${ }^{7}$ See e.g. E. Wirsing, Elementare Beweise des Primzahlsatzes mit Restglied. II, J. Reine Angew. Math. 214/215 (1964), 1-18. 
A better result is known, ${ }^{6}$ but it is interesting to notice that this can be proved by elementary means since (5) can. ${ }^{7}$

Faculté des Sciences d'Orsay, France

\section{SOME PROPERTIES OF INTEGRAL CLOSURE}

\section{WILLIAM J. HEINZER}

Let $D$ be an integrally closed domain with identity having quotient field $K$, let $L$ be an algebraic extension field of $K$, and let $\bar{D}$ be the integral closure of $D$ in $L$. We prove here that the following five ideal theoretic structure properties of $\bar{D}$ are inherited by $D$, namely: (a) $\bar{D}$ is a Prüfer domain, (b) $\bar{D}$ is an almost Dedekind domain, ${ }^{1}$ (c) $\bar{D}$ is a Dedekind domain, (d) $\bar{D}$ has the $Q R$-property, ${ }^{2}$ (e) $\bar{D}$ has property (\#). ${ }^{3}$

The converse of (a) is true (that is, $D$ Prüfer implies that $\bar{D}$ is Prüfer) and was established by Prüfer in $[11$, p. 31]. In case $L$ is finite-dimensional over $K$, Noether [9, p. 37] proved the converse of (c) and Butts and Phillips [2, p. 270] proved the converse of (b). In the general case it is well-known or easy to see that the converses of (b), (c) and (e) are false. The converse of (d) is false (see [6, p. 102]) even when $L$ is finite-dimensional over $K$.

Our statements concerning (a), (b) and (c) will be obtained as corollaries to the following.

Theorem 1. Let $M$ be a prime ideal of $\bar{D}$ and let $P=M \cap D$. Then $\bar{D}_{M} \cap K=D_{P}$.

Proof. It is clear that $D_{P}$ is contained in $\bar{D}_{M} \cap K$. To obtain the reverse inclusion we first observe that $L$ may be assumed to be a normal extension of $K$. For let $E$ be a normal closure of $L$ over $K, D^{*}$ the integral closure of $\bar{D}$ in $E$, and $N$ a prime ideal of $D^{*}$ lying over $M$. Then $N \cap D=P$ and $\bar{D}_{M} \subseteq D_{N}^{*} \cap L$ so that $\bar{D}_{M} \cap K \subseteq D_{N}^{*} \cap K$. Let $\left\{M_{\alpha}\right\}$ be the set of prime ideals of $\bar{D}$ lying over $P$. By a well-known

Received by the editors July 26, 1966.

${ }^{1} D$ is almost Dedekind if for each maximal ideal $P$ of $D, D_{P}$ is a discrete rank one valuation ring $[4$, p. 813$]$.

${ }^{2} D$ has the $Q R$-property if each integral domain between $D$ and its quotient field is a quotient ring of $D[6$, p. 97].

${ }^{3} D$ is said to have property (\#) if for $\Delta_{1}$ and $\Delta_{2}$ distinct subsets of the set of maximal ideals of $\bar{D}$ we have $\bigcap_{M \in \Delta_{1}} D_{M} \neq \bigcap_{M \in \Delta_{2}} D_{M}[5$, p. 331]. 